\title{
ADNP-CSMA Random Multiple Access protocol application with the function of monitoring in Ad Hoc network
}

\author{
Gang ZHAN ${ }^{1}$,Yi Fan ZHAO ${ }^{1}$,Hong Wei DING ${ }^{1, \text { a }}$,Sheng Jie ZHOU ${ }^{1}$, Long Jun LIU $^{1}$ and Zhi XU ${ }^{1}$ \\ ${ }^{1}$ Institute of Information, Yunnan University, Kunming, Yunnan, 650504, China
}

\begin{abstract}
In Ad Hoc networks, the net work of mobile nodes exchange information with their wireless transceiver equipment,the network throughput is in increased,compared to other such multiple hops network.Moreover along with the rapid development of modern information,communication business also will be increase.However,the access and adaptive of previous CSMA protocol are insufficient.According to these properties,this paper presents a kind of adaptive dual clock with monitoring function P-CSMA random multiple access protocol(ADNP-CSMA), and discusses two kinds of P-CSMA.ACK with monitoring function is introduced to maintain the stability of the whole system,and the introduction of dual clock mechanism reduces the channel of idle period.It calculate the system throughput expression through the method of average period,and the simulation results show that the system is constant in the case of high load throughput.
\end{abstract}

Keywords: Adaption; Dual Clock; The monitoring function; CSMA;Throughput

\section{Introduction}

With the rapid development of wireless technology, the dynamic nature of the network node draws pubic attention,thus it arises Ad Hoc network at the historic moment.It makes up the limited transmission distance with the help of technology for wireless devices through jumping forward,and it hosts networking communication freely through the moving mobile.It provides the successful solution for military communication,disaster relief and temporary communication[1].

MAC layer channel access protocol directly affects the performance of the whole system.How to use the channel effectively becomes the key issues of the whole network.We adopt a new kind of CSMA protocol to research this problem.[2]

There are three kinds of traditional CSMA, N-CSMA, 1-CSMA and P-CSMA.These three protocols have some shortcoming.P-CSMA is defined as pointing $\mathrm{P}$ probability to send in idle 
time, and retreating in the probability of 1-P.When a packet arrives in busy time,there is two cases following:P-insist in idle period with non-insist in busy period CSMA and P-insist in idle period with 1-insist in busy period CSMA.Paper 2 discusses P-insist in idle period with 1-insist in busy period CSMA,and we call it 1P-CSMA.When the $\mathrm{P}$ equals to 1 ,it becomes 1-insist CSMA.We discuss the protocol in the circumstance of P-insist in idle period with non-insist in busy period CSMA, and we called it NP-CSMA.[3]

We need the data of each node,however,the nodes only send data in the access channel,and it can not know that the data is transmitted to the right recipient.If it adds a monitoring information section(ACK) at the end of each packet,then we called it P-CSMA protocol with the function of monitoring.After adding a monitoring information, the system will be able to know about the sending information group and channel.The monitoring information plays an oversight role, and this is an important improvement.It obtain a monitoring of the whole system with little throughput loss.[4]

The system uses discrete clock to control channel.If arriving a packet,it begins to send in next sequence.If arriving a packet in idle period,it also begins to send in next sequence of idle period,thus it wastes the channel utilization.If it uses continuous clock control in idle period. When it arrives a packet in a sequence of idle period,the packet do not have to wait for the beginning of the sequence to send,then sends immediately.Such control principle reduces the average length of idle period,and increases the average length of the transmission period.It is helpful to increase the throughput of the whole system.[5]

\section{The analysis of the proposed protocol}

\subsection{The analysis of $A C K$ mechanism}

The channel of P-CSMA with ACK random multiple access protocol is shown in Figure 1.[6]

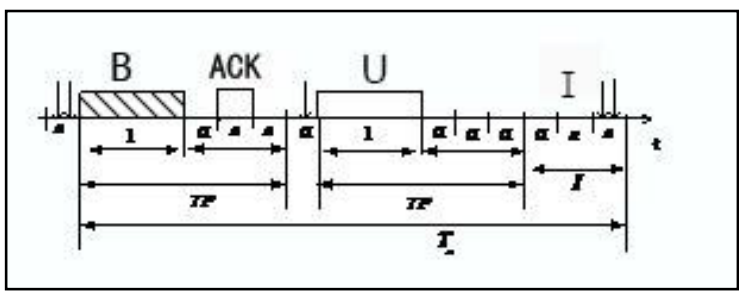

Figure.1 The channel of P-CSMA with ACK random multiple access protocol

The transfer cycle divided into three parts, the packet reached 1,transmission delay a, and the ACK with the function of monitoring

In this model,there will be three random events:

Event that information packets are sent successfully( $U$ events).

Event that information packets collide with each other(the collision appears, $B$ events).

Event that there are no information packets in the channel arrive,the channel is idle( $I$ events).[7]

Supposing we put the $B$ events and $U$ events as $B U$ events,so the channel appears on two event, $I$ events and $B U$ events(we called busy events).

Before the mathematical analysis of channel,we make the following assumptions:[8]

1.The channel access method is MAC control protocol of random multiple access,and there will be independent of process parameters for $G$ poisson process;

2.The length of idle time sequence is a in the channel,and the length of sending packet time sequence is 1 ;

3.The channel is assumed to be the ideal state, and no noise channel;

4.The collision packet will be retransmitted at a certain moment,and the retransmitted packet has no effect on channel arrival process;

5.The length of ACK with the monitoring function is $\tau_{A}\left(\tau_{A}=a\right)$; 
In a idle period,the probability of no reaching packet in the channel is:

$$
q_{0}^{0}=e^{-a p G}
$$

In a idle period,the probability of a reaching packet in the channel is:

$$
q_{0}^{1}=a p G e^{-a p G}
$$

In a cycle,the probability of continuous $i$ idle events is:

$$
P\left(N_{I}=i\right)=\left(e^{-a p G}\right)^{i}
$$

In a cycle,the probability of continuous $j$ $B U$ events is:

$$
P\left(N_{B U}=j\right)=\left(1-e^{-a p G}\right)^{j}
$$

In a cycle,the probability of continuous $i I$ events and $j B U$ events is:

$$
P\left(N_{I}=i, N_{B U}=j\right)=\left(e^{-a p G}\right)^{i}\left(1-e^{-a p G}\right)^{j}
$$

The averages length of $i$ continuous $j$ events in a cycle is:

$E(I)=a \sum_{i=1}^{n} \sum_{j=1}^{n} i p\left(N_{I}=i, N_{B U}=j\right)=\frac{a}{1-e^{-a p G}}$

(6)

The averages length of $j$ continuous $B U$ events in a cycle is:

$$
\begin{aligned}
& E(B U)=\left(1+2 a+\tau_{A}\right) \sum_{i=1}^{n} \sum_{j=1}^{n} j p\left(N_{I}=i, N_{B U}=j\right) \\
& =\frac{1+2 a+\tau_{A}}{e^{-a p G}}
\end{aligned}
$$

The averages length of information packet transmitted successfully is:

$$
E(U)=\frac{q_{0}^{1}}{1-q_{0}^{0}}=\frac{\operatorname{ap} G e^{-a p G}}{1-e^{-a p G}}
$$

The throughput of P-CSMA random multiple access protocol with ACK mechanism is:

$$
S=\frac{E(U)}{E(I)+E(B U)}
$$

$$
\begin{aligned}
& =\frac{\frac{a p G e^{-a p G}}{1-e^{-a p G}}}{\frac{a}{1-e^{-a p G}}+\frac{\left(1+2 a+\tau_{A}\right)}{e^{-a p G}}} \\
& =\frac{a p G\left(e^{-a p G}\right)^{2}}{a e^{-a p G}+\left(1+2 a+\tau_{A}\right)\left(1-e^{-a p G}\right)}
\end{aligned}
$$

\subsection{The analysis of dual}

\section{clocks mechanism}

When the system is idle,the system uses continuous-time control strategy.And if the system is busy,the system uses discrete-time control strategy.[9]

The period of idle time sequences obeys the geometric distribution of the mean:

$$
E[N]=\frac{1}{1-e^{-a p G}}
$$

the normalized probability of a reaching packet in a time sequence is:

$$
P_{I_{1}}=\frac{a p G e^{-a p G}}{1-e^{-a p G}}
$$

The normalized probability of two or more reaching packets in a time sequence is:

$$
P_{I_{2}}=\frac{1-a p G e^{-a p G}-e^{-a p G}}{1-e^{-a p G}}
$$

Because the reaching pockets in idle period will interrupt the idle period,so we can take the middle point of the mean. The averages length of $I$ events is:

$$
\begin{aligned}
& E(I)=\left(\frac{1}{1-e^{-a p G}}-1\right) a+\frac{a^{2} p G e^{-a p G}}{2\left(1-e^{-a p G}\right)}+\frac{\left(1-a p G e^{-a p G}-e^{-a p G}\right) a}{2\left(1-e^{-a p G}\right)} \\
& =\frac{\left(1+e^{-a p G}\right) a}{2\left(1-e^{-a p G}\right)}
\end{aligned}
$$

The throughput of the dual clocks P-insist CSMA random multiple access protocol with $\mathrm{ACK}$ mechanism is:

$$
S=\frac{E(U)}{E(I)+E(B U)}
$$

$$
=\frac{\frac{a p G e^{-a p G}}{1-e^{-a p G}}}{\frac{a\left(1+e^{-a p G}\right)}{2\left(1-e^{-a p G}\right)}+\frac{1+2 a+\tau_{A}}{e^{-a p G}}}
$$




$$
=\frac{2 a p G\left(e^{-a p G}\right)^{2}}{a e^{-a p G}\left(1+e^{-a p G}\right)+2\left(1-e^{-a p G}\right)\left(1+2 a+\tau_{A}\right)}
$$

\subsection{The analysis of the adaptive mechanism}

The above is throughput expression.In order to realize the adaptive function,we need to adjust the size of the $P$ according to the system load value,namely to $P$ derivative:[8]

$$
\frac{d S}{d P}=0
$$

Due to that getting for nonlinear equations,we obtain approximate solution by adopting the method of Newton type.

When the condition of light load $G \in(0,2]$, the probability $P$ is 1 . When the load increases slowly, probability $P$ also corresponding reduces. We get the approximate solution of the probability $P$ by adopting the piecewise idea to obtain the maximum throughput case. Supposing the relationship between $P$ and $G$ is:

$$
P=\frac{1}{\beta \times G}
$$

$P$ and $G$ combination cases can be drawn from $\beta$ value:

$$
\left.P\right|_{M A X}= \begin{cases}1 & G \in(0,2] \\ \frac{1}{0.5 G} & G \in(2,3] \\ \frac{1}{0.333 G} & G \in(3,5] \\ \frac{1}{0.2 G} & G \in(5,8.5] \\ \frac{1}{0.1 G} & G \in(8.5, \infty)\end{cases}
$$

\section{Simulation results and analysis}

With the simulation tool MATLAB R2014a, the simulation results are shown in Figure 2 to
5 , in case $\mathrm{a}=0.1$.

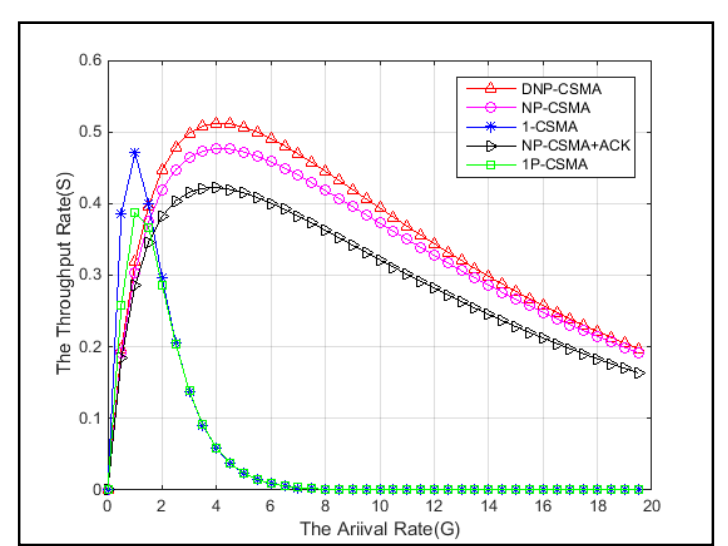

Figure.2 The comparison of several different CSMA

In the Fig.2,compared to DNP-CSMA, NP-CSMA, 1-CSMA,NP-CSMA with ACK, P-CSMA, when $\mathrm{P}=0.5,1 \mathrm{P}-\mathrm{CSMA}$ reached the maximum throughput rate at 1.8 G,but NP-CSMA reached the maximum throughput rate at 4 G.Moreover NP-CSMA maximum throughput rate is bigger than 1P-CSMA.When $\mathrm{G}=6$, 1P-CSMA throughput rate is 0,but NP-CSMA throughput rate would be 0 after 20 G.DNP-CSMA reduced idle time sequence,so the throughput rate increases a bit. NP-CSMA with ACK adds the monitoring fields,so the throughput rate decreases a bit.

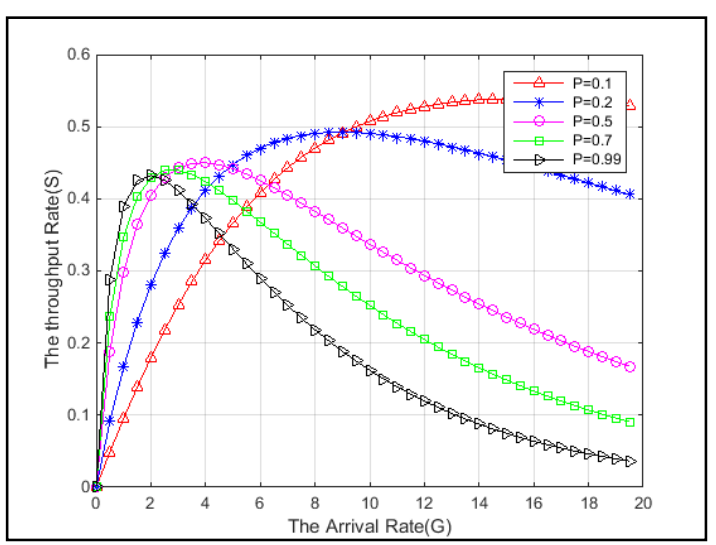

Figure.3 The throughput rate of DNP-CSMA with different $P$

In the Fig.3, it can be seen that DNP-CSMA in different $P$ effects the throughput rate of the system.

With the increase of $P$, the probability of success that the sites send information packets 
in idle channel also grows,and the channel utilization increases, so the throughput rate of the system increases.However with the rapid of information packets arrival rate $G$, the system throughput rate also fells rapidly,finally tends to zero.

So we can improve performance of the system by controlling $P$. If we find the critical point, it will reach maximum throughput rate of the system.Then this reminds us of the adaptive mechanism.

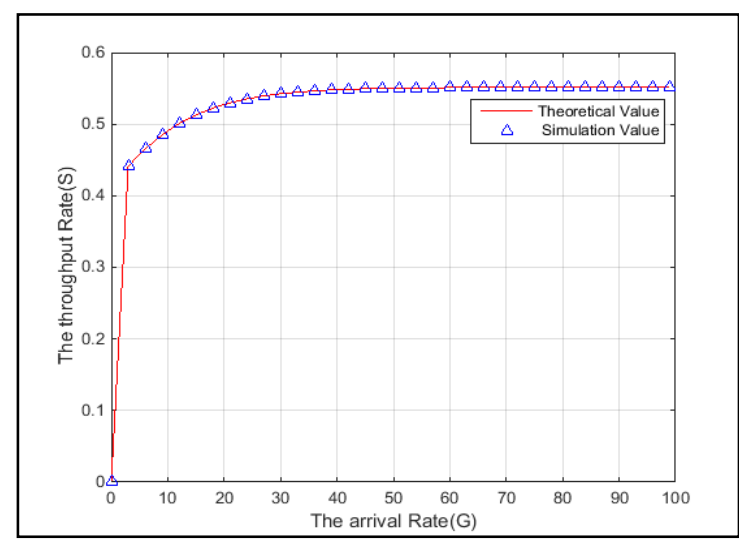

Figure.4 The throughput rate of ADNP-CSMA

In the Fig.4,after adding the adaptive mechanism, the system throughput rate is stable in arrival rate $G$ reaching 30,and then with the increase of load, the system throughput is to maintain a constant value,so the system turns into a steady state.

In the adaptive system, the probability $\mathrm{P}$ in the whole process can be dynamic adjustment according to the circumstance of network load to make the optimal throughput rate.This mechanism can ensure that the throughput rate can still maintain large under the heavy load network.

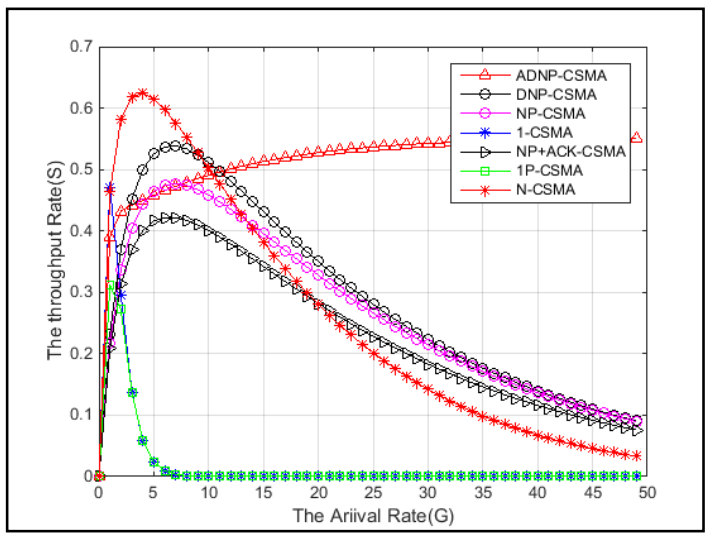

Figure.5 The comparison of several different CSMA when $\mathrm{P}=0.3$

In the Fig.5,compared to ADNP-CSMA, DNP-CSMA, NP-CSMA,1-CSMA,NP-CSMA with ACK, P-CSMA,N-CSMA,the throughput rate of ADNP-CSMA is better than NP-CSMA's and 1P-CSMA's. For $\mathrm{N}$-CSMA, although in case of light load, the throughput rate of N-CSMA is higher than ADNP-CSMA's, however, with the arrival rate increasing N-CSMA's throughput rate reduces, finally turns zero,and ANDP-CSMA's throughput rate is stabilize after arrival rate $G$ is 30. Therefore, the new protocol for network has more advantages in heavy load.

\section{Conclusions}

In this paper, ADNP-CSMA protocol is proposed on the basis of traditional CSMA protocol, which distinguishes between NP-CSMA and 1P-CSMA. Moreover the Dual Clock mechanism and the function of monitoring mechanism are put forward, increasing the throughput rate and maintaining the stability of the system. After introducing the adaptive function, the system can dynamic adjustment to send probability $\mathrm{P}$ according to the weight of the load. It is ensure that the system can still maintain high throughput rate in case of heavy load,and it improves the performance of the wireless communication system. At the same time, the simulation experimental results show the consistency and the rationality of the theoretical analysis and 
the simulation experiment. Compared to the throughput rate of different systems, ADNP-CSMA random multiple access protocol system has more advantages in the case of heavy load.

\section{References}

1. George, Y., and I. Bergel. "The Spectral Efficiency of Slotted CSMA Ad-Hoc Networks with Directional Antennas." IEEE Transactions on Wireless Communications 11.10(2012):3799-3809.

2. Dongfeng Zhao. Study on A New Method for Continuous-time Systems of Random Access Channel [J]. Journal of Electronics, 21(1): 37-41, 1999.

3. Dongfeng Zhao, Study on A New Method for the Slotted Access Channel [J], Journal of Electronics,Vol.19, pp.814-819, No.6,1997.

4. Hongwei Ding, Yingying Guo, Yifan Zhao, Shengjie Zhou, and Qianlin Liu. Research on the Multi-Channel Probability Detection CSMA Protocol with Sensor Monitoring Function. Sensor Lett. 13, 143-146 (2015).

5. Maomao Huang. The analysis of time-sharing CSMA wireless sensor network MAC control protocol analysis[D]. Kunming: Yunan University 2009

6. Dongfeng Zhao. Study on the Average Cycle Method for S-ALOHA Access Channel[J].Journal of Yunnan University, 1994, 16(2): pp133-138.

7. Liu Binbin. The Analysis of Multi-channel Random Multiple Access Wireless Communication Network Protocol based on Probability Detection [D]. Kunming: Yunnan University, 2006.

8. Shengjie Zhou et al., Research on the Discrete time Three-Dimensional Probability Csma Protocol In ad-hoc Network. International Journal of Recent
Scientific Research Vol. 6, Issue, 5, pp.4257-4262, June, 2015.

9. Sheng Jie Zhou, Hong Wei Ding, Meng Yao Wang, et al. Multi-channel Dual Clocks three-dimensional probability Random Multiple Access protocol for Wireless Public Bus Networks based on RTS/CTS mechanism[J]. MATEC Web of Conferences, 2016, 44. 